\title{
Buffer composition affects rose bengal dialysis rate through cellulose membrane
}

BIOOPEN 2021 - POST-CONFERENCE ARTICLE

\section{KRZYSZTOF SZTANDERA (D)}

University of Lodz, Faculty of Biology and Environmental Protection, Department of General Biophysics, Pomorska 141/143, 90-236 Lodz, Poland

E-mail: krzysztof.sztandera@edu.uni.lodz.pl

\section{Abstract}

Due to its fluorescent and phototoxic properties, rose bengal (RB) is used in photodynamic therapy. To improve the delivery of $\mathrm{RB}$ to its site of action, the application of nanocarrier systems has been proposed. The most promising approach includes the use of $\mathrm{pH}$-responsive nanoparticles. To evaluate the pattern of drug release in different buffers, equilibrium dialysis is commonly used. Here, we used water and two buffers to determine the impact of solvent composition on the aggregation and dialysis rate of $\mathrm{RB}$ through a cellulose membrane. The results show that buffer composition does not influence the fluorescent properties of RB. However, the presence of additional ions causes a change in diffusion rate that is most probably linked to the size of RB aggregates.

KEYWORDS: nanocarriers, dialysis, rose bengal, photodynamic therapy.

\section{Introduction}

Rose bengal (RB) is one of the synthetic dyes, designed as a fluorescein analogue and classified as a xanthene (Snyder and Paugh 1998). It is used in the textile (Alexander 2010), food (Cossu et al. 2016) and pharmaceutical industries (Patel et al. 2020). In medicine, RB is used for staining damaged conjunctival and corneal cells to indicate eye damage (Bron et al. 2003) and for diagnosis of liver and eye cancer (Capinera and Squitier 2000) as well as (due to the fluorescent properties) as a photosensitizer in anticancer photodynamic therapy (Qin et al. 2017). RB is characterized by maximum absorbance at $550 \mathrm{~nm}$, high efficiency of free radical production, fluorescence emission, low photodegradation, non-toxicity in the dark and a lack of allergic reactions and other side effects (Allison et al. 2004). However, its applicability may be limited due to the hydrophilic nature of RB, leading to its poor cellular uptake (Gianotti et al. 2014). Thus, the main advantage of nanotechnology in this case is based on increasing transmembrane transport of the drug, leading to an increase of its photodynamic potential (Sztandera et al. 2020). Nowadays nanotechnology 
allows the synthesis of complex nanocarriers such as dendrimers (Dabrzalska et al. 2017), polymersomes (Villani et al. 2017) or dendrimersomes (Apartsin et al. 2020). Depending on the type of nanoparticle, $\mathrm{RB}$ can be conjugated via a linker to the surface of the nanoparticle or encapsulated inside its structure (Patri et al. 2005). To limit potential side effects, a photosensitizer should be released from its nanocarrier under strictly controlled conditions occurring only in neoplastic lesions. $\mathrm{pH}$ is probably the best factor for triggering the release in the case of anticancer therapies, due to the slightly acidic tumour environment (Sztandera et al. 2019). Evaluating this feature is crucial during the design of new nanosystems. For these assays and for the purpose of purification steps, in which free $\mathrm{RB}$ is removed from the nanocarrier solution, equilibrium dialysis is most frequently used. Application of semi-permeable membranes with an optimal weight cut-off allows the separation of molecules with different sizes, and the use of different $\mathrm{pH}$ conditions enables an evaluation of $\mathrm{pH}$-dependent release of the drug from its nanocarrier. However, since different nanoparticles and carrier systems require different buffers, which may affect the properties of the therapeutics and dialysis membranes, it is important to evaluate the correlation between buffer composition and the rate of $\mathrm{RB}$ dialysis through the membrane.

\section{Materials and methods}

\section{Materials}

RB and PBS were purchased from Sigma-Aldrich (Taufkirchen, Germany). Snakeskin $^{\mathrm{TM}}$ Dialysis Tubing $(3.5 \mathrm{~K}$ MWCO, $22 \mathrm{~mm}$ ) was obtained from Thermo Fisher Scientific (Waltham, MA, USA). $\mathrm{Na}_{2} \mathrm{HPO}_{4}$ and $\mathrm{NaH}_{2} \mathrm{PO}_{4}$ required to prepare phosphate buffer were purchased from $\mathrm{POCH}$ (Gliwice, Poland).

\section{Spectroscopy studies}

Fluorescence spectra were acquired using a PerkinElmer LS-50B spectrofluorometer. Measurements of the evaluated RB samples were performed in proper buffer, at room temperature. The excitation wavelength was set to $525 \mathrm{~nm}$ and spectra were collected in a wavelength range from 540 to $640 \mathrm{~nm}$. Excitation and emission slits were 5 and $7 \mathrm{~nm}$, respectively.

\section{Dialysis}

To evaluate the rate of photosensitizer dialysis against different buffers, a $50 \mu \mathrm{M}$ solution of $\mathrm{RB}$ was enclosed in dialysis membrane tubing (SnakeSkin ${ }^{\mathrm{TM}}$ Dialysis Tubing, 3.5K MWCO, $22 \mathrm{~mm}$; Thermo Fisher) and immersed in PBS (10 mM, pH 7.4), phosphate buffer (10 mM, pH 7.4) or $\mathrm{H}_{2} \mathrm{O}$. Samples from the internal phase were collected at subsequent intervals $(0.5,1,2,4,8$ and $24 \mathrm{~h}$ ) and measured spectrophotometrically using a PerkinElmer LS-50B spectrofluorometer.

\section{Size measurements}

Size measurements were performed using a Zetasizer Nano ZS (Malvern Instruments Ltd., UK) at a constant temperature of $25^{\circ} \mathrm{C}$ to check the hydrodynamic diameter of $\mathrm{RB}$ immersed in different buffers. Samples at the final concentration of $\mathrm{RB}(100 \mu \mathrm{M})$ were prepared in PBS (10 mM, pH 7.4), phosphate buffer (10 mM, pH 7.4) and $\mathrm{H}_{2} \mathrm{O}$.

\section{Statistical analysis}

Two-way ANOVA for concentration series followed by post-hoc Tukey's test for pairwise difference testing were used to test statistical significance. In all tests, p-values $<0.05$ were considered statistically significant. Data are presented as arithmetic mean $\pm \mathrm{SD}$. 


\section{Results}

Fluorescence measurements showed that the maximum fluorescence intensity of $\mathrm{RB}$ occurs at $565 \mathrm{~nm}$ regardless of the buffer used (Figure 1). Moreover, the fluorescence intensity of RB was almost the same in all examined buffers.

Based on the fluorescence intensity from the previous experiment, we calculated the percentage of $\mathrm{RB}$ remaining in the dialysis tube after a specified time (Figure 2). RB diffused through the cellulose membrane over time; however, the release rate was dependent on the type of external buffer. Dialysis against PBS was the most rapid - after $6 \mathrm{~h}$ almost all RB molecules were released. Diffusion in phosphate buffer was slower; however, the release rate after $24 \mathrm{~h}$ was like that in PBS. By contrast, dialysis against water was very slow and after 24 h only $20 \%$ of RB was released from the dialysis tube.

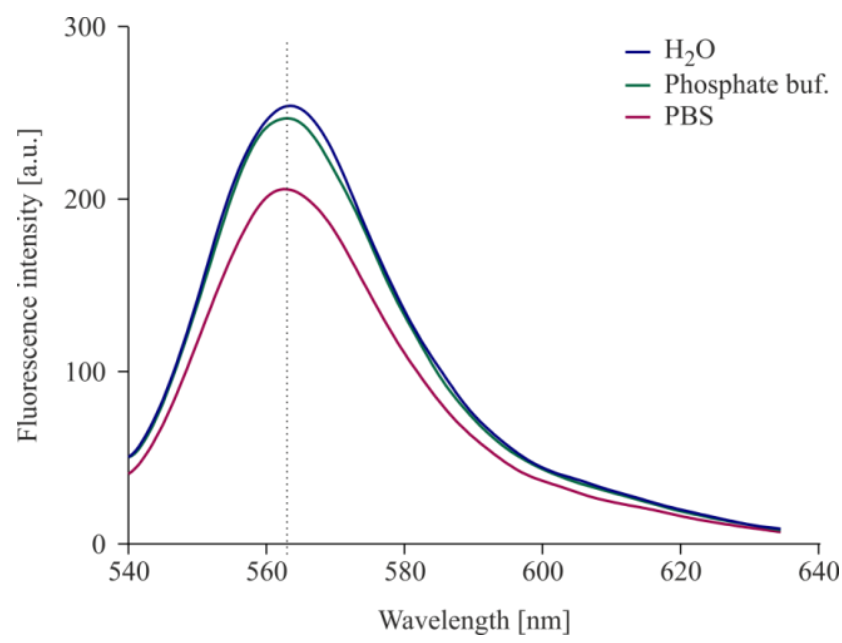

Figure 1. Fluorescence spectra of $\mathrm{RB}(1 \mu \mathrm{M})$ in $\mathrm{PBS}, \mathrm{H}_{2} \mathrm{O}$ and phosphate buffer.

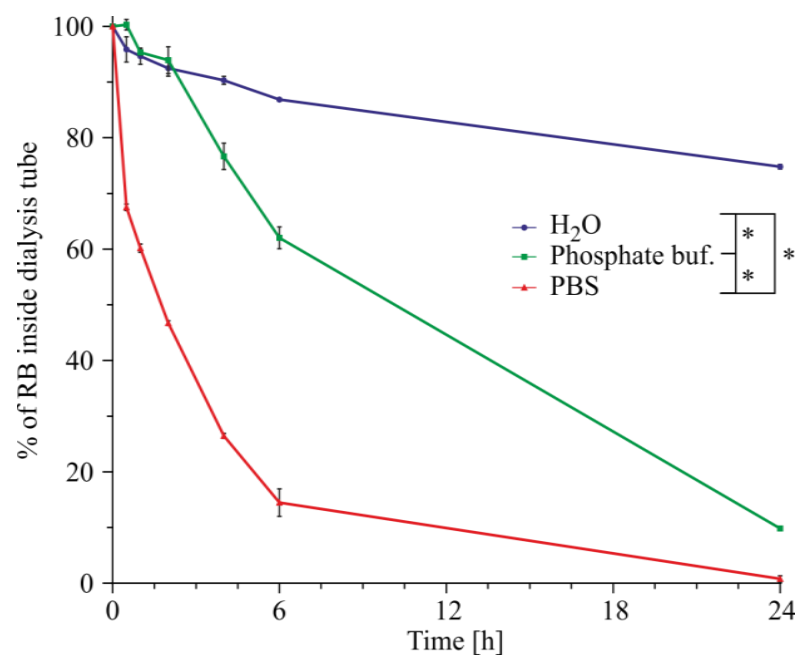

Figure 2. Dialysis of RB against different buffers. Data presented as mean $\pm \mathrm{SD},{ }^{*} \mathrm{p}<0.05$. 
Table 1. Hydrodynamic diameter of RB dissolved in evaluated buffers.

\begin{tabular}{|l|c|c|c|}
\hline & $\mathbf{H}_{2} \mathbf{O}$ & Phosphate buffer & PBS \\
\hline $\begin{array}{l}\text { Hydrodynamic } \\
\text { diameter [nm] }\end{array}$ & $120.76 \pm 37.25$ & $1.27 \pm 0.32$ & $0.72 \pm 0.13$ \\
\hline
\end{tabular}

Measurements of hydrodynamic diameter showed that the composition of the buffer influences the aggregation of RB. The largest RB aggregates were observed in $\mathrm{H}_{2} \mathrm{O}$, while in PBS and phosphate buffer there were almost no clumps observed.

\section{Discussion}

Equilibrium dialysis is a relatively easy but very important step in the preparation and characterization of new drugs and their carriers. Owing to the application of semi-permeable membranes, this method allows the purification of final products from residues and checking of the conditions of drug release from nanosystems (Apartsin et al. 2020; Wu et al. 2013). Our experiments have shown that buffer composition does not cause a shift of the maximum emission wavelength of $\mathrm{RB}$, but greatly impacts the diffusion rate of this dye through semi-permeable cellulose membrane. Dialysis buffers used in the experiments differed in their ion content: water was ion-free, phosphate buffer contained sodium and phosphate ions, and PBS in addition to these also contained chloride and potassium ions in high concentrations (Cold Spring Harbor Protocols 2006). In the case of dialysis, buffers have an additional advantage as opposed to water - they are able to maintain a constant $\mathrm{pH}$, which is critical in the case of $\mathrm{pH}$-sensitive nanosystems (Karimi et al. 2016). Based on hydrodynamic diameter measurements, we can conclude that the composition of buffer influences RB aggregation, which results in the presence of $\mathrm{RB}$ in the solution in the form of aggregates of various sizes (largest in water, smallest in PBS buffer, most probably due to interactions of the anionic RB with oppositely charged ions in the solution). A cellulose membrane has a so-called molecular weight cut-off (MWCO), which is the largest particle size that can pass through the membrane. Thus, smaller molecules diffuse more rapidly than particles with a size close to the MWCO, which is reflected in our results - the aggregates in water could not cross the membrane as fast as those in phosphate buffers. Moreover, in a production process, a membrane with heterogenous pores is usually obtained, which may additionally slow down the dialysis rate (Haney et al. 2013). Finally, one additional possible explanation involves changing the properties of hydrophilic cellulose membrane in the presence of counter-ions and facilitating the passage of solutes.

\section{Conclusions}

Buffer composition significantly influences the diffusion of RB through a semi-permeable cellulose membrane. The most probable explanation involves the influence of ions contained in the buffers that protect RB from clumping compared to the environment of ion-free water, where the biggest aggregates were observed. Increased size of RB aggregates limits dialysis efficiency. Thus, the optimization of this method is crucial to prevent miscalculation of the concentration of final product or misstatement about the $\mathrm{pH}$-dependent stability/lability of bonding with nanoparticles. 


\section{References}

Alexander, W. 2010. American Society of Clinical Oncology, 2010 Annual Meeting and rose bengal: from a wool dye to a cancer therapy. Pharmacy and Therapeutics, 35: 469-474.

Allison, R.R., Downie, G.H., Cuenca, R., Hu, X.H., Childs, C.J.H., Sibata, C.H. 2004. Photosensitizers in clinical PDT. Photodiagnosis and Photodynamic Therapy 1(1): 27-42.

Apartsin, E., Knauer, N., Arkhipova, V., Pashkina, E., Aktanova, A., Poletaeva, J., SánchezNieves, J., de la Mata, F.J., Gómez, R. 2020. $\mathrm{pH}$-sensitive dendrimersomes of hybrid triazine-carbosilane dendritic amphiphilessmart vehicles for drug delivery. Nanomaterials 10(10): 1-15.

Bron, A.J., Evans, V.E., Smith, J.A. 2003. Grading of corneal and conjunctival staining in the context of other dry eye tests. Cornea 22(7): 640-650.

Capinera, J.L., Squitier, J.M. 2000. Insecticidal activity of photoactive dyes to American and migratory grasshoppers (Orthoptera: Acrididae). Journal of Economic Entomology 93(3): 662-666.

Cold Spring Harbor Protocols. 2006. Phosphatebuffered saline (PBS). Cold Spring Harbor Protocols 2006(1): pdb.rec8247.

Cossu, A., Ercan, D., Tikekar, R.V., Nitin, N. 2016. Antimicrobial effect of photosensitized rose bengal on bacteria and viruses in model wash water. Food and Bioprocess Technology 9(3): 441-451.

Dabrzalska, M., Janaszewska, A., Zablocka, M., Mignani, S., Majoral, J.P., Klajnert-Maculewicz, B. 2017. Cationic phosphorus dendrimer enhances photodynamic activity of rose bengal against basal cell carcinoma cell lines. Molecular Pharmaceutics 14(5): 1821-1830.

Gianotti, E., Estevão, B.M., Cucinotta, F., Hioka, N., Rizzi, M., Renò, F., Marchese, L. 2014. An efficient rose bengal based nanoplatform for photodynamic therapy. Chemistry - A European Journal 20(35): 10921-10925.

Haney, P., Herting, K., Smith, S. 2013. Separation characteristics of dialysis membranes. Pierce previews. Pierce Protein Biology Products, 2 -6 .

Karimi, M., Eslami, M., Sahandi-Zangabad, P., Mirab, F., Farajisafiloo, N., Shafaei, Z., Ghosh, D., Bozorgomid, M., Dashkhaneh, F., Hamblin,
M.R. 2016. pH-sensitive stimulus-responsive nanocarriers for targeted delivery of therapeutic agents. Wiley Interdisciplinary Reviews: Nanomedicine and Nanobiotechnology 8(5): 696-716.

Patel, S.P., Carter, B.W., Murthy, R., Sheth, R., Agarwala, S.S., Lu, G., Redstone, E., Balmes, G.S., Rider, H., Rodrigues, D., Wachter, E.A. 2020. Percutaneous hepatic injection of rose bengal disodium (PV-10) in metastatic uveal melanoma. Journal of Clinical Oncology 38(15 suppl): 3143.

Patri, A.K., Kukowska-Latallo, J.F., Baker, J.R. 2005. Targeted drug delivery with dendrimers: comparison of the release kinetics of covalently conjugated drug and non-covalent drug inclusion complex. Advanced Drug Delivery Reviews 57(15): 2203-2214.

Qin, J., Kunda, N., Qiao, G., Calata, J.F., Pardiwala, K., Prabhakar, B.S., Maker, A.V. 2017. Colon cancer cell treatment with rose bengal generates a protective immune response via immunogenic cell death. Cell Death and Disease 8(2): e2584.

Snyder, C., Paugh, J.R. 1998. Rose bengal dye concentration and volume delivered via dyeimpregnated paper strips. Optometry and Vision Science 75 (5): 339-341.

Sztandera, K., Działak, P., Marcinkowska, M., Stańczyk, M., Gorzkiewicz, M., Janaszewska, A., Klajnert-Maculewicz, B. 2019. Sugar modification enhances cytotoxic activity of PAMAM-doxorubicin conjugate in glucosedeprived MCF-7 cells - possible role of GLUT1 transporter. Pharmaceutical Research 36(10): 1-11.

Sztandera, K., Gorzkiewicz, M., Klajnert-Maculewicz, B. 2020. Nanocarriers in photodynamic therapy - in vitro and in vivo studies. Wiley Interdisciplinary Reviews: Nanomedicine and Nanobiotechnology 12(3): 1-24.

Villani, S., Adami, R., Reverchon, E., Ferretti, A.M., Ponti, A., Lepretti, M., Caputo, I., Izzo, L. 2017. pH-sensitive polymersomes: controlling swelling via copolymer structure and chemical composition. Journal of Drug Targeting 25(9-10): 899-909.

Wu, H., Zhu, L., Torchilin, V.P. 2013. pH-sensitive poly(histidine)-PEG/DSPE-PEG co-polymer micelles for cytosolic drug delivery. Biomaterials 34(4): 1213-1222. 